\title{
The maternal-neonatal neuro-immune interface: Are there long-term implications for inflammatory or stress-related disease?
}

\author{
Nola Shanks and Stafford L. Lightman \\ University Research Centre for Neuroendocrinology, University of Bristol, Dorothy Hodgkins-Crowfoot Laboratories, \\ Bristol, United Kingdom \\ Address correspondence to: Nola Shanks, University Research Centre for Neuroendocrinology, University of Bristol, \\ Dorothy Hodgkins-Crowfoot Laboratories, Marlborough Street, Bristol, BS2 8HW United Kingdom. \\ Phone: 44-117-928-3450; Fax: 44-117-928-3399; E-mail: N.Shanks@bristol.ac.uk.
}

J. Clin. Invest. 108:1567-1573 (2001). DOI:10.1172/JCI200114592.

Inflammatory stimuli reliably elicit hypothalamic-pituitary-adrenal (HPA) activation, and it is now established that the immune and HPA systems are mutually regulatory and that their interactions partly determine stress effects on immune function. What has not been extensively investigated, however, is the interactive nature of the development of the endocrine and immune systems and whether this might alter predisposition to inflammatory disease or stress-related pathologies. Increasing attention is now being focused on the role of early life environments determining longterm predisposition to disease, and a role for the HPA axis in disease predisposition is emerging.

Stress generally refers to the condition where coping with actual or perceived stimuli alters the homeostatic state of the organism. The classic stress response involves the activation of central and peripheral catecholamine systems and HPA responses. Increased glucocorticoid levels are thought to protect the organism, both by providing energy substrates to deal with immediate environmental demands and by blocking potentially harmful overreaction by the immune system and other stress-reactive systems (1). However, to avoid immunosuppression and degenerative pathologies associated with increased glucocorticoid levels, the HPA stress response must terminate efficiently once the environmental demands are removed (2). Consequently, disturbances in endocrine-immune interactions upset the normal regulatory homeostatic balance and alter susceptibility to a variety of disease states associated with immune dysregulation (3).

The physiological impact of stress is variable across individuals, and susceptibility to stress apparently depends on attributes of the organism, such as genetic endowment and age, and experiential factors, such as previous stress experiences, and early life events $(4,5)$. Indeed, the endocrine and immune systems and the CNS all respond to environmental pressures during development, and the interactive nature of these systems suggests that alterations in one system could have developmental consequences for the others and potential long-term implications for disease vulnerability. Plasticity in most developing physiological systems is thought to be of adaptive importance, enabling an organism to better meet the demands of the environment in which it matures and will most likely reside. However, the short-term reorganization or re-setting of regulatory systems to deal with immediate demands may also have long-term consequences for normal physiological function, thereby predisposing an organism to pathology. This may particularly be the case when the environmental pressures differ between development and adulthood.

A number of epidemiological studies have indicated that nutritional factors early in life have long-term implications for cardiovascular and metabolic disease in adulthood. Most data suggest that maternal nutrition and limited intrauterine growth are responsible for reorganizing metabolic regulation and results in metabolic and cardiovascular pathologies in adult life (6). The concept of "perinatal programming" is not new and has long been investigated in neuroendocrine development of both the adrenal and the gonadal axes (see review, ref. 7). Immune responses, too, are influenced by early life experiences, and it has been suggested that pathogen exposure during development is important for the developing immune repertoire and that too little activation may be associated with the development of allergy (8). Available data also indicate that there are long-term consequences following endocrine-immune interactions during the first week of life. Thus, the administration of steroids during antigen exposure alters the development of immune tolerance (9), and immune challenge during the first week of life is associated with altered endocrine development $(10,11)$. These data demonstrate the interactive nature of both the immune and the neuroendocrine systems during development and show that these interactions can alter both immune and endocrine function in the long term. 
Maternal factors represent some of the most salient environmental influences altering endocrine development (12-14). Indeed, there is an extensive literature detailing both prenatal and postnatal influences on the development of the HPA axis. It is also well established that immune factors passed in utero and also to the neonate via milk have a direct impact on the its immunocompetence $(15,16)$. However, evidence examining the neuro-immune interface and maternal factors is limited.

\section{Prenatal maternal influences}

Alterations in the neuro-immune interface begin in utero, and prenatal stress exposure in mid- to late pregnancy has been associated with increased HPA stress responsivity in offspring (see review, ref. 14). However, the impact of prenatal stress on immune function is complex and varies depending on the stage of pregnancy at which the animal is stressed, the sex of the offspring, the species, and, indeed, what immune compartment and response one considers. Consequently, no general statement concerning the impact of prenatal stress on the offspring's immunocompetence is possible.

Treatment of pregnant female primates at mid- to late pregnancy with adrenocorticotropic hormone (ACTH) to stimulate the HPA axis diminishes IL- 6 and febrile responses to IL-1 $\beta$ administration in 1.5- to 2 -year-old offspring (17), indicating that sufficient activation of the HPA axis during pregnancy can exert long-term effects on cytokine and inflammatory responses. Prenatal stress has also been shown to disrupt placental transfer of IgG in squirrel monkeys, although this effect depends on the sex of the offspring. Male monkeys are born with lower-than-normal IgG levels, and females are born with higher-than-normal levels that also exceed those of the mother (16). Prenatal stress effects also appear to vary with the stage of pregnancy at which disruption occurs. Disruption during early pregnancy increases cellular immune responses, whereas prenatal stress exposure or dexamethasone treatment during mid- to late pregnancy can be immunosuppressive in adult offspring (17). Taken together, these data indicate that, at least in primates, there is considerable plasticity in the developing neuroendocrine-immune system (17). Furthermore, prenatal stress, or, more specifically, activation of the HPA axis, exerts long-term effects on immune regulation in offspring and may have considerable implications for predisposition to infectious and autoimmune disease later in life.

In rodent models, prenatal stress is generally administered during the final trimester, and this is when the most notable alterations in HPA function are observed (14). Gorczynski (18) examined conditioned stress effects on offspring when stress was administered to pregnant dams late in pregnancy. Using a cross-fostering design to determine whether effects on the offspring depend upon the treatment of the biological mother or upon postnatal factors consequent to the stress treatment, Gorczynski observed that stress-related immunosuppression (as measured by antibody responses and skin graft rejection) was most pro- nounced in offspring born and fostered to prenatally stressed dams. Interestingly, offspring of nonstressed dams and then fostered to stressed dams also exhibited immunosuppression relative to control animals, implying that prenatal stress effects are mediated both in utero through the feto-placental unit and postnatally either through alterations in maternal behavior or via milk-borne factors. Exposure of pregnant dams to footshock stress, psychological stress, and noise stress has all been shown to alter IgG levels in juvenile rats (19), and prenatal noise stress exposure has also been shown to reduce arthus skin reactions and delayed-type hypersensitivity reactions in offspring. Adult rat offspring of prenatally stressed dams also exhibit increased antibody response to antigen, although prenatal stress effects are less marked for natural killer (NK) cell cytotoxicity and are not observed for splenocyte proliferative responses to mitogens (20). These data are further confirmed by observations of Kay et al. (21) that prenatal stress exposure reduces NK cell cytotoxicity in both the spleen and the blood but that these effects are less pronounced for splenocyte responses to phytohemagglutinin. Interestingly, these researchers also observed a marked reduction in the splenic B cell proliferative responses to pokeweed mitogen in prenatally stressed animals and showed that these effects could not be explained by cellular migration between immune compartments. What appears to be consistent across both primate and rodent data is that both antibody responses and inflammation are particularly susceptible to prenatal stress effects. Indeed, prenatal stress accelerates the onset and increases the prevalence of diabetes in the nonobese diabetic (NOD) mouse model of insulin-dependent diabetes (22). Overall, the rodent data confirm what has been reported for primate models and suggest that prenatal stress can alter predisposition to pathology.

The bidirectional influence of immune and endocrine factors raises important questions about the impact of prenatal immune activation on the developing neuroimmune interface. While limited, the data on this point are particularly interesting. Prenatal immunization with a T-dependent antigen or with Gram-negative LPS increases basal levels of circulating corticosterone in adult offspring and decreases glucocorticoid and mineralocortioid receptor binding levels in CNS structures that are important for negative feedback regulation of the HPA axis (23). Administration of antigen can increase ACTH and corticosterone responses to a stress (23). Administration of IL- $1 \beta$ to pregnant females has been reported to have long- as well as short-term effects on developing endocrine systems. Thus, Gotz et al. (24) have found that sexual behaviors in adult male offspring, locomotor activity, and learning are all markedly affected and that corticosterone responses are blunted following exposure to a novel stressor. Cytokines produced in response to maternal infection might mediate the endocrine effects on developing offspring, as increased levels of proinflammatory cytokines have been observed in the placenta, amniotic fluid, and fetal brain following maternal challenge with LPS (25). These 
data suggest that maternal infection or immune activation has long-term implications for CNS organization and developing endocrine systems. Indeed, it has even been suggested that the impact of maternal immune factors may contribute in some way to psychiatric disease, and it appears that this might particularly be the case for disorders with HPA involvement.

\section{Postnatal environment and maternal factors}

When considering postnatal stimulus saliency, maternal interactions with offspring are paramount. Interactions between the mother and offspring are complex, and not only are they important for meeting the nutritional requirements of the offspring, they also provide warmth and tactile stimulation. Considerable literature exists regarding the importance of maternal contact on the psychological development of primates; however, more recent attention has focused on how important maternal and peer contact are for the developing endocrine (12) and immune systems (see review, ref. 26). Mitogen-induced lymphocyte proliferation is reduced in monkeys that as infants were subjected to early separation (i.e., maternal or peer). In-depth analyses of the effects of early rearing environments on immune functioning reveal that 2-year-old rhesus monkeys that had been reared in a nursery have reduced $\mathrm{CD}^{+}$cell numbers and lowered NK cell cytotoxicity, but increased mitogen-induced lymphocyte proliferation up to 2.5 years of age, relative to controls that were reared by their mothers (26). Monkeys that had been separated on multiple occasions from their mothers early in life also showed long-term suppression of cell-mediated immune responses. However, the data also differentiate between the impact of rearing conditions and that of intermittent maternal separation. It is difficult to confirm whether increased immune responses seen in offspring reared under nursery conditions might also reflect differences in pathogen exposure early in life compared with mother-raised infants. It is also uncertain whether the increase in cell-mediated activity reflects the removal of maternal influences or stress. Analysis of the immediate impact of maternal separation on lymphocyte proliferation in infant primates reveals that immune disturbances are most notable in those infants that displayed greater behavioral responses to the separation procedure. Over a 14-day separation, mitogen-induced lymphocyte proliferation responses drop significantly relative to preseparation base-line levels in infant bonnet monkeys. Levels return to base line once these infants are reunited with their mothers (26). In addition to exogenous in vitro measures of immune activity, maternal separation has also been shown to disrupt the normal balance of intestinal microflora in infant rhesus monkeys (27). Those infants displaying the strongest emotional reactions to separation were found to display the greatest drop in microflora and to be more susceptible to opportunistic bacterial infections. Maternal separation thus appears to be stressful for primates and to alter the risk of disease both immediately during and after the separation and far into adulthood.
Rodent models investigating the importance of maternal factors in development of endocrine and immune systems have largely used maternal separation/deprivation or neonatal handling paradigms. What is interesting about these neonatal manipulations is that they exert opposing effects on HPA function. "Handling" involves separation of the dam from the nest for 15-20 minutes per day. Following handling sessions, dams generally exhibit increased nesting bouts and manipulation of offspring, and it has been suggested that this increase in maternal behavior is the determining factor mediating the long-term effects of handling on development of the HPA axis and stress responsiveness (12). This observation is further supported by data indicating that HPA stress responses to maternal separation in rat pups can be altered by tactile stimulation (28). Stroking rat pups with a brush while they are separated from their mother dampens ACTH responses, while feeding alters both corticosterone and ACTH responses (28). These data suggest that maternal interactions with pups during early development represent more than maintenance of nest temperature and provision of milk, i.e., that manipulation of offspring and maternal behaviors are important factors that can modulate the impact of early life stress. Maccari and colleagues (29) elegantly demonstrate the importance of maternal behavior in determining endocrine development in a study where offspring from prenatally stressed dams were cross-fostered/adopted to other dams. These authors found that, regardless of the prenatal stress condition, cross-fostering increased maternal interactions with offspring, reversing the effects of prenatal stress on HPA stress responsivity. Consequently, it appears that plasticity, at least with respect to HPA development in rodents, extends to the first weeks of life and that postnatal events can have opposing effects to prenatal pressures on developing neuroendocrine systems.

As adults, neonatally handled animals generally exhibit dampened HPA stress responses, and greater negative feedback regulation of HPA responses than nonhandled animals (see ref. 5). Neonatal handling in mice enhances cell-mediated immune responses such as NK cell cytotoxicity and mitogen-induced lymphocyte proliferation in adult mice (30). In contrast, differences in immune responsiveness between handled and nonhandled rats were not as pronounced or have not been consistently observed for either antibody responses or predisposition to tumors $(31,32)$. However, the long-term effects of handling on neuro-immune interactions become more obvious when the animals are stressed. Thus, following exposure to either acute cold stress or chronic intermittent cold stress, the plaqueforming response to sheep erythrocytes is decreased to a similar extent for both handled and nonhandled animals (31). If, however, the chronically stressed animals are re-exposed to the stressor, immune responses are suppressed even further in the nonhandled animals, but no further suppression is seen for the handled animals (31), suggesting that while antibody responses are suppressed in the chronically stressed handled animals, 
they are better able to contend with subsequent stress exposure than are nonhandled animals.

The effects of neonatal handling with respect to susceptibility to disease are more difficult to interpret. Handling increases susceptibility to experimental allergic encephalomyelitis (EAE), increasing both incidence and severity of the clinical signs of the pathology (33). In addition to dampening HPA responses, handling may promote long-lasting alterations in the cellular and molecular environments in the brain that increase susceptibility to EAE in adult life (34). However, neonatal handling does not appear to alter susceptibility to another autoimmune condition, adjuvant-induced arthritis (AA) (11). It might be expected that animals with lower HPA activity would prove more susceptible or exhibit more severe inflammation, but circulating levels of corticosterone and altered peptide expression within the paraventricular nucleus of the hypothalamus in response to the AA inflammation are similar in handled and nonhandled animals, a finding that may account for the similar symptom profiles observed between these two experimental groups (11).

In contrast to the neonatal handling effects, adult animals maternally deprived or exposed to a longer duration of maternal separation during development generally display increased HPA responses to stress as adults (see review, ref. 13) and decreased plaque-forming cell responses. Laban et al. (35) have noted that maternal deprivation exerts the opposite effects on susceptibility to EAE, where both the neurological and the histological signs of the inflammation are suppressed compared with those in control animals. However, these researchers also observed that early weaning of pups exacerbated both the clinical signs and the histological lesions of EAE. Maternal factors and early life stress may, therefore, interact and alter long-term predisposition to inflammation as a consequence of increased HPA activity.

Administration of dexamethasone to neonatal rats has also been found to exert long-term effects changing susceptibility to inflammatory disease. Bakker and colleagues (36) injected rat pups with dexamethasone or saline during the first week of life. When the animals were 8 weeks of age, the researchers assessed the HPA responses to acute inflammatory challenge (i.e., an LPS injection), macrophage cytokine production, and induced EAE. They observed that animals administered dexamethasone as neonates had significantly lower plasma corticosterone responses to LPS challenge and that macrophages from these animals produced lower levels of inflammatory cytokines, whereas splenocytes expressed greater increased levels of mRNA for IFN- $\gamma$ and TNF- $\beta$. Interestingly, these animals exhibited increased incidence and severity of EAE relative to saline treated controls. These researchers also suggest that exposure to glucocorticoid during early development may have implications for intrathymic $\mathrm{T}$ cell selection events resulting in long-term changes in immune reactivity. These data further illustrate the interactive nature of the endocrine and immune systems during development and that exposure to syn- thetic glucocorticoids can exert permanent programming effects on the neuro-immune interface.

In addition to behavioral variables, milk-borne factors should not be ruled out as having an important role in the development of neuro-immune interactions. Lactation is unique to the mammalian species and not only serves to provide nutrition to offspring but plays an important role in the development of immune and possibly endocrine function. Both breast milk and colostrum are complex matrices containing hormones, lymphocytes, antibodies, and cytokines. These immune and endocrine signals are transferred to offspring and are thought to prepare the developing organism to deal with environmental challenges and pathogens. Glucocorticoids and other steroids present in milk are important for the offspring's normal respiratory, gut, endocrine, and CNS development. Corticosterone administered to lactating dams can be passed to offspring and elevate circulating steroid levels in rat pups (37). As adults, these animals show dampened corticosterone stress responses, increased central steroid receptor levels, and altered behavior. The permanent alterations seen in these animals are strikingly similar to profiles seen in neonatally handled animals, and it could be suggested that mild stress due to handling procedures might provoke maternal HPA responses and thus contribute to handling effects on development of offspring. The observation that maternal glucocorticoids influence endocrine development in offspring also suggests that postnatal maternal stress could alter CNS, endocrine, and possibly immune development. Postnatal maternal immune activity may similarly alter development of progeny. In particular, induction of EAE prior to pregnancy or during lactation reduced susceptibility to EAE in offspring (38). These effects do not depend on the disease severity in the dams, and the greatest protection is seen for animals where the dam was immunized shortly after delivery, when the refractory period was during lactation. Only pups nursed by immunized dams were protected, whether born to immunized or nonimmunized dams. Protection therefore does not appear to be related to antibody passively transferred to the neonate, suggesting that other immune or even endocrine factors transferred via colostrum or milk can affect susceptibility to inflammation over the long term.

Most of the attention concerning long-term effects of early life environment has been on how endocrine or behavioral factors influence the development of the immune system and neuroendocrine regulation of immune function. However, given the bidirectional communication between the two systems, stimuli that elicit an immune response may similarly program the developing CNS and endocrine systems. Administration of IL-1 $\beta$ to mouse pups during the first week of life has been shown to alter corticosterone and ACTH levels in adult mice and to increase splenocyte responses to allogeneic antigens (39). Neonatal rats treated with a low dose of endotoxin during the first week of life exhibit increased HPA stress responsiveness as adults (10). Adult animals that had been administered endotoxin 
during development show greater corticosterone and ACTH stress responses, perhaps reflecting both increased drive of the HPA axis by increased hypothalamic peptide release and less efficient negative feedback inhibition consequent to lower glucocorticoid receptor levels in the CNS (10). Basal secretion patterns are also increased in adult animals that have been exposed to endotoxin as neonates, and these animals exhibit increased anxiety and behavioral stress reactivity (11).
However, the most striking feature of these animals is that, as adults, they do not develop paw inflammation subsequent to induction of AA, although, like control animals, their levels of corticosterone increase and hypothalamic corticotropin-releasing hormone $(\mathrm{CRH})$ and arginine vasopressin (AVP) mRNA expression are altered following adjuvant administration (11). Exposure to acute inflammatory stimuli during development, while obviously altering HPA regulation and

Table 1

Summary of the evidence examining neuroendocrine-immune interactions during development and their impact on immune and endocrine function

$$
\text { Event Neuroendocrine-immune alterations }
$$

Prenatal

Endocrine $\rightarrow$ Immune

Primates

ACTH mid-/late gestation

Psychological stress mid-/late gestation

Stress mid-/late gestation

Stress early gestation

Dexamethasone/Stress mid-/late gestation

Dexamethasone mid-/late gestation

Rodents Mice Conditioned stress late gestation

Rats

Stress late gestation

Stress late gestation

Stress late gestation

Stress late gestation

Stress late gestation

Immune $\rightarrow$ Endocrine

Rodents

Rats

LPS exposure late gestation

Inoculation with human erythrocytes IL-1 $\beta$ exposure

Postnatal

Endocrine $\rightarrow$ Immune

Primates

Separation (maternal/peer)

Nursery rearing

Frequent maternal separation

Rodents Rats

Maternal deprivation/separation

Early weaning

Maternal EAE during lactation

Dexamethasone treatment

Mice Neonatal handling

Rats Neonatal handling

Immune $\rightarrow$ Endocrine

Rodents Rats

Exposure to IL-1 $\beta$

Exposure to LPS $\downarrow$ IL-6, $\downarrow$ IL-1 $\beta$-induced febrile response

NC IL- 6 or IL-1 $\beta$ febrile response

IgG transfer $\downarrow$ male offspring, $\uparrow$ female offspring

$\uparrow$ Cellular immune response (mixed lymphocyte response)

$\downarrow$ Cellular immune response (mixed lymphocyte response)

$\downarrow$ Mitogen-induced lymphocyte proliferation (ConA)

$\downarrow$ Lymphocyte sensitivity to steroid in vitro

$\downarrow$ Antibody response to sheep erythrocytes

$\downarrow$ Skin/graft rejection

$\uparrow$ Prevalence and acceleration of onset of diabetes in NOD mice

$\downarrow$ IgG levels in neonatal offspring

$\downarrow$ Antibody titers to herpes simplex $1, \downarrow$ arthus skin reaction

$\downarrow$ Delayed-type hypersensitivity

NC mitogen-induced splenocyte proliferation

Marginal decrease in NK cell cytotoxicity, $\uparrow$ antibodies to antigen

$\downarrow$ NK cell cytotoxicity, $\downarrow$ splenocyte proliferation to PWM

$\uparrow$ Basal corticosterone,

$\downarrow$ Mineralocorticoid and glucocorticoid CNS receptor binding

$\uparrow$ Corticosterone and ACTH stress responses

$\uparrow$ Activity, $\downarrow$ cognitive performance, $\downarrow$ stress corticosterone

$\downarrow$ Mitogen-induced proliferation

$\downarrow$ Intestinal microflora

$\downarrow$ CD $8^{+}$cells, $\downarrow$ NK cell cytotoxicity, $\uparrow$ mitogen proliferation

$\downarrow$ Mitogen-induced lymphocyte proliferation

$\downarrow \mathrm{EAE}$

$\uparrow \mathrm{EAE}$

$\downarrow$ EAE in offspring

$\uparrow \mathrm{EAE}, \uparrow$ splenocyte proinflammatory cytokine mRNA

$\uparrow N K$ cell cytotoxicity, $\uparrow T$ cell mitogenesis

No difference between basal and acute or chronic stress effects on

plaque-forming cell response to sheep erythrocytes, $\uparrow$ sensitivity

of response to stress re-exposure

$\downarrow$ Number of DMBA-induced tumors and $\uparrow$ survival time $\quad 32$

$\uparrow$ EAE

NC paw inflammation for $A A$

$\downarrow$ Morning basal corticosterone

39

$\uparrow$ Corticosterone and ACTH stress responses

$\downarrow$ CNS glucocorticoid receptor binding

$\uparrow$ Hypothalamic CRH and AVP mRNA expression

$\uparrow$ Basal corticosterone

$\downarrow$ AA inflammation

$\uparrow$, Increase; $\downarrow$, decrease; NC, no change; ConA, concanavalin A; DMBA, 7,12-dimethylbenz(a)anthracene; PWM, pokeweed mitogen. 


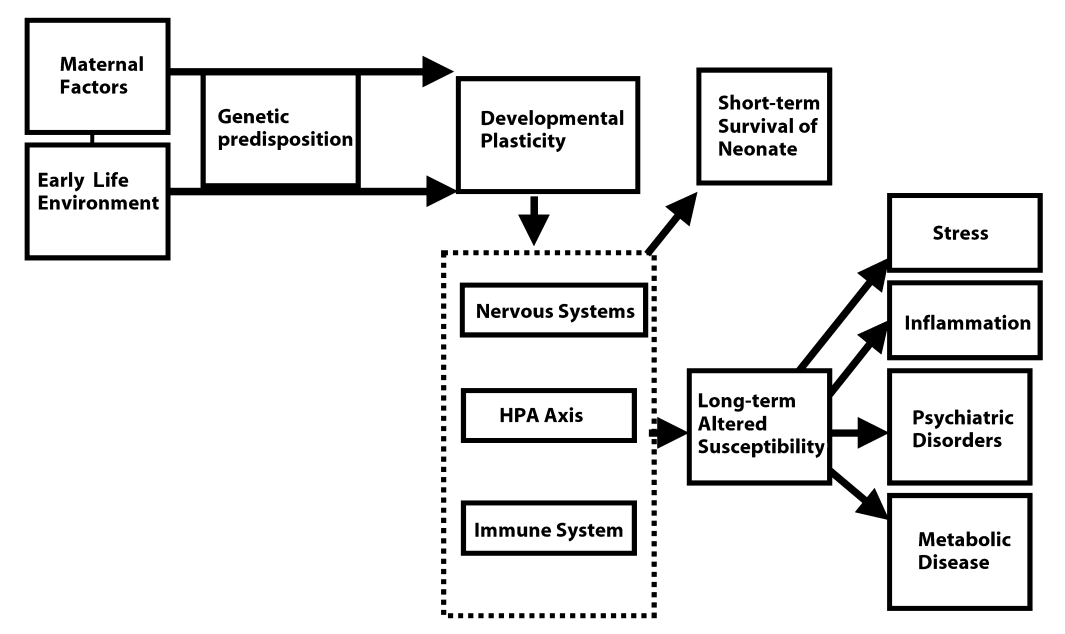

Figure 1

Schematic representation of the impact of maternal and early life environment factors altering neuroendocrine development. Developmental plasticity, which allows the organism to contend with environmental pressures to survive as a neonate, may alter predisposition to disease over the long-term.

stress responsiveness, thus appears to also alter the regulation of inflammation by neuroendocrine factors. Consistent with this view, splenocyte proliferation to LPS is more sensitive to stress factors when the cells are collected from adult animals that had been neonatally exposed to endotoxin. Corticosterone responses to intravenous injection of LPS are also accelerated in these animals relative to controls (11).

It is apparent, therefore, that both prenatal and postnatal environmental factors can influence HPA development, alter stress responsiveness, and alter predisposition to inflammation. Predisposition to inflammation appears to be tightly linked to the responsivity of the HPA axis. While regulation of the onset of inflammation and predisposition to autoimmune disease are multifaceted, it has been suggested that in adult animals immune factors (e.g., tolerance) affect disease susceptibility, whereas HPA responsiveness dictates disease severity $(4,40)$. However, as is apparent from the developmental literature, endocrine activity can alter the developing immune system and might, therefore, also dictate disease vulnerability.

\section{A lifelong homeostatic balance}

Evidently, maternal factors play a fundamental role mediating the impact of environmental pressures on developing neonatal physiology (see Table 1). However, while some of the data are contradictory, they do consistently indicate that maternal factors influence the developing fetus and extend to perinatal programming of physiological responses. Why these mechanisms are in place, and the significance of developmental plasticity of the neuro-immune interface, are cause for speculation. If we are to believe that these interactions are important for maintaining a homeostatic balance for the developing organism, interference with these processes should jeopardize survival. In fact, this is clearly illustrated when animals fail to mount an adequate glucocorticoid response to an acute inflammatory challenge. HPA responses may be necessary to suppress inflammatory immune responses to septic challenges and thereby combat septic shock, effectively protecting the organism from its own immune response. We would expect that an animal that is faced with repeated immune challenges during development would, therefore, benefit from increased HPA responsiveness.

What, if any, therapeutic indications can be gained from the observations that endocrine-immune interactions during development can exert permanent alterations in physiological function and predisposition to disease? The data suggest that the immune system may represent novel therapeutic avenues in populations predisposed to endocrine or psychiatric anomalies, and that, alternatively, early life stressors may have implications for susceptibility to inflammation later in life. Interactions between immune and endocrine systems display tremendous plasticity and exhibit functional alterations that extend into adulthood; however, a longterm cost may be incurred subsequent to the immediate adaptation seen during the pre- and perinatal periods (see Figure 1). While it might be advantageous for the developing organism to mount a prolonged glucocorticoid response to control inflammatory responses and to have increased immune responsiveness to HPA regulation/stress factors, the cost of permanent alterations in HPA responsivity may be stress-related disease or pathologies with HPA components. In particular, increased HPA activity might prove detrimental for those individuals susceptible to metabolic diseases or psychiatric diseases such as depression, both known to be stress-related and influenced by elevated glucocorticoid levels. Indeed, the role of early life infections or inflammation has now been recognized in epidemiological research and is beginning to be confirmed in clinical studies as associated with respiratory disease and coronary heart disease secondary to metabolic disease. It is not clearly established, however, whether early life infections are associated with increased incidence of psychiatric disease; but there is increasing evidence that there are immunoregulatory perturbations associated with depression (see review, ref. 41).

Do prenatal and early life stress and HPA activity, then, have implications for immune regulation or dysregulation in adulthood? Kavelaars and colleagues (42) have extended their work, initially done on rodents, that suggests that perinatal glucocorticoid treatment 
has side effects and alters immune function through to adulthood. They have demonstrated that $T$ cell proliferation responses are suppressed in term and preterm infant cord blood following perinatal glucocorticoid treatment. While the long-term implications of these effects have yet to be established, preliminary data suggest that in humans there are neuro-immune interactions in utero that might have implications for postnatal immunocompetence. If there are long-term consequences of prenatal glucocorticoid exposure, this could call into question existing practices of administering glucocorticoids during pregnancy to stimulate the development of the fetal lung.

The maternal-neonatal neuro-immune interface represents a unique window of developmental plasticity where detection of environmental perturbations can lead to permanent alterations in physiological function for the organism. Activation of the HPA axis and immune factors such as proinflammatory cytokines represent potential mechanisms transmitting environmental information to developing systems that are exquisitely sensitive to these inputs. These environmental inputs then alter the developmental course of "responsive" systems such as the endocrine, CNS, and immune systems, creating an organism programmed to meet particular environmental demands. Pathology is then thought to result when environmental demands in adulthood no longer match the physiological adjustments made earlier in life. Early life factors may thus contribute to individual vulnerability to stress-related disease and to inflammation in adulthood.

1. Stein, M., and Miller, A.H. 1993. Stress, the hypothalamic-pituitary-adrenal axis, and immune function. Adv. Exp. Med. Biol. 335:1-5.

2. McEwen, B.S., and Stellar, E. 1993. Stress and the individual: mechanisms leading to disease. Arch. Intern. Med. 153:2093-2101.

3. Biondi, M., and Zannino, LG. 1997. Psychological stress, neuroimmunomodulation, and susceptibility to infectious disease in animals and man: a review. Proc. Natl. Acad. Sci. USA. 93:3043-3047.

4. Mason, D. 1991. Genetic variation in the stress response: susceptibility to experimental allergic encephalomyelitis and implications for human inflammatory disease. Immunol. Today. 12:57-60.

5. Meaney, M.J., et al. 1994. Early environmental programming hypothalamic-pituitary-adrenal responses to stress. Seminars in the Neurosciences. 6:247-259.

6. Barker, D.J.P. 1992. The fetal origins of disease. J. Hypertens. 10(Suppl. 7):S39-S44.

7. Matsumoto, A., and Arai, Y. 1997. Sexual differentiation of neuronal circuitry in the neuroendocrine hypothalamus. Biomed. Rev. 7:5-15.

8. Rook, G.A., and Stanford, J.L. 1998. Give us this day our daily germs. Immunol. Today. 19:113-116.

9. O'Grady, M.P., and Hall, N.R.S. 1991. Long-term effects of neuroendocrineimmune interactions during early development. In Psychoneuroimmunology. 2nd edition. R. Ader, D.L. Felten, and N. Cohen, editors. Academic Press Inc. San Diego, California, USA. 561-572.

10. Shanks, N., Larocque, S., and Meaney, M.J. 1995. Neonatal endotoxin exposure alters the development of the hypothalamic-pituitary-adrenal axis: early illness and later responsivity to stress. J. Neurosci. 15:376-384.

11. Shanks, N., et al. 2000. Early-life exposure to endotoxin alters hypothalamic-pituitary-adrenal function and predisposition to inflammation. Proc. Natl. Acad. Sci. USA. 97:5645-5650.

12. Liu, D., et al. 1997. Maternal care, hippocampal glucocorticoid receptors, and hypothalamic-pituitary-adrenal responses to stress. Science. 277:1659-1662.

13. Kaufman, J., Plotsky, P.M., Nemeroff, C.B., and Charney, D.S. 2000. Effects of early adverse experiences on brain structure and function: clinical implications. Biol. Psychiatry. 48:778-790.

14. Wellberg, L.A.M., and Seckl, J.R. 2001. Prenatal stress, glucocorticoids and the programming of the brain. J. Neuroendocrinol. 13:113-128.

15. Coe, C.L., and Crispen, H.R. 2000. Social stress in pregnant squirrel monkeys (Saimiri boliviensis peruviensis) differentially affects placental transfer of maternal antibody to male and female infants. Health Psychol. 19:554-559.

16. Coe, C.L., and Lubach, G.R. 2000. Prenatal influences on the neuroimmune set points in infancy. Ann. NY Acad. Sci. 917:468-477.

17. Reyes, T.M., and Coe, C.L. 1997. Prenatal manipulations reduce the proinflammatory response to a cytokine challenge in juvenile monkeys. Brain Res. 769:29-35.

18. Gorczynski, R.M. 1992. Conditioned stress responses by pregnant and/or lactating mice reduce immune responses of their offspring after weaning. Brain Behav. Immun. 6:87-95.

19. Sobrian, S.K., et al. 1997. Gestational exposure to loud noise alters the development and postnatal responsiveness of humoral and cellular components of the immune system in offspring. Environ. Res. 73:227-241.

20. Klein, S.L., and Rager, D.R. 1995. Prenatal stress alters immune function in the offspring of rats. Dev. Psychobiol. 28:321-336.

21. Kay, G., Tarcic, N., Poltyrev, T., and Weinstock, M. 1998. Prenatal stress depresses immune function in rats. Physiol. Behav. 63:397-402.

22. Saravia-Fernandez, F., Durant, S., el Hasnaoui, A., Dardenne, M., and HomoDelarche, F. 1996. Environmental and experimental procedures leading to variation in the incidence of diabetes in the nonobese diabetic (NOD) mouse. Autoimmunity. 24:113-121.

23. Reul, J.M., et al. 1994. Prenatal immune challenge alters the hypothalamicpituitary-adrenocortical axis in adult rats. J. Clin. Invest. 93:2600-2607.

24. Gotz, F., et al. 1993. Short- and long-term effects of perinatal interleukin-1 beta-application in rats. Neuroendocrinology. 58:344-351.

25. Urakubo, A., Jarskog, L.F., Lieberman, J.A., and Gilmore, J.H. 2001. Prenatal exposure to maternal infection alters cytokine expression in the placenta, amniotic fluid, and fetal brain. Schizophr. Res. 47:27-36.

26. Worlein, J.M., and Laudenslager, M.L. 2001. Effects of early rearing experiences and social interactions on immune function in nonhuman primates. In Psychoneuroimmunology. 3rd edition. Volume 2. R. Ader, D.L. Felten, and N. Cohen, editors. Academic Press Inc. San Diego, California, USA. 73-85.

27. Bailey, M.T., and Coe, C.L. 1999. Maternal separation disrupts the integrity of the intestinal microflora in infant rhesus monkeys. Dev. Psychobiol. 35:146-155.

28. Rosenfeld, P., Suchecki, D., and Levine, S. 1992. Multifactorial regulation of the hypothalamic-pituitary-adrenal axis during development. Neurosci. Biobehav. Rev. 16:553-568.

29. Maccari, S., et al. 1995. Adoption reverses the long-term impairment in glucocorticoid feedback induced by prenatal stress. J. Neurosci. 15:110-116.

30. Neveu, P.J., Deleplanque, B., Puglisis-Allegra, S., D’Amato, F.R., and Cabib, S. 1994. Influence of early life events on immune reactivity in adult mice. Dev. Psychobiol. 27:205-213.

31. Bhatnagar, S., Shanks, N., and Meaney, M.J. 1996. Plaque-forming cell response and antibody-titers following injection of sheep red-blood cells in nonstressed, acute and/or chronically stressed handled and nonhandled animals. Dev. Psychobiol. 29:171-181.

32. Hilakivi-Clarke, L., Wright, A., and Lippman, M.E. 1993. DMBA-induced mammary tumor growth in rats exhibiting increased or decreased ability to cope with stress due to early postnatal handling or antidepressant treatment. Physiol. Behav. 54:229-236.

33. Laban, O., Dimitrijevic, M., von Hoersten, S., Markovic, B.M., and Jankovic, B.D. 1995. Experimental allergic encephalomyelitis in adult DA rats subjected to neonatal handling or gentling. Brain Res. 676:133-140.

34. Manni, L., Micera, A., Pistillo, L., and Aloe, L. 1998. Neonatal handling in EAE-susceptible rats alters NGF levels and mast cell distribution in the brain. Int. J. Dev. Neurosci. 16:1-8.

35. Laban, O., Markovic, B.M., Dimitrijevic, M., and Jankovic, B.D. 1995. Maternal deprivation and early weaning modulate EAE in the rat. Brain Behav. Immun. 9:9-19.

36. Bakker, J.M., et al. 2000. Neonatal dexamethasone treatment increases susceptibility to experimental autoimmune disease in adult rats. J. Immunol. 165:5932-5937.

37. Catalani, A., et al. 2000. Maternal corticosterone during lactation permanently affect brain corticosteroid receptors, stress response and behaviour in rat progeny. Neuroscience. 100:319-325.

38. Dimitrijevic, M., Markovic, B.M., Laban, O., and Jankovic, B.D. 1995. Suppression of experimental allergic encephalomyelitis in offspring of DA and Wistar rats following immunisation of mother with encephalitogen. J. Neuroimmunol. 58:43-50.

39. del Rey, A., et al. 1996. Alterations in the pituitary-adrenal axis of adult mice following neonatal exposure to interleukin-1. Brain Behav. Immun. 10:235-248.

40. van de Lagerijt, A.G., et al. 1994. Susceptibility to adjuvant arthritis: relative importance of adrenal activity and bacterial flora. Clin. Exp. Immunol. 97:33-38.

41. Kronfol, Z., and Remick, D.G. 2000. Cytokines and the brain: implications for clinical psychiatry. Am. J. Psychiatry. 157:683-694.

42. Kavelaars, A., et al. 1999. Altered immune function in human newborns after prenatal administration of betamethasone: enhanced natural killer cell activity and decreased $\mathrm{T}$ cell proliferation in cord blood. Pediatr. Res. 45:306-312. 